\title{
Severity of foot process effacement is associated with proteinuria in patients with IgA nephropathy
}

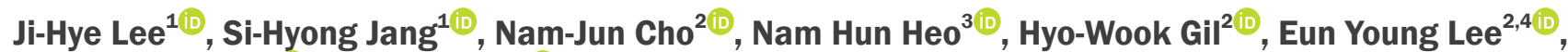 \\ Jong-Seok Moon ${ }^{5(i)}$, Samel Park ${ }^{2,5}$ \\ 'Department of Pathology, College of Medicine, Soonchunhyang University, Cheonan, Republic of Korea \\ ${ }^{2}$ Department of Internal Medicine, Soonchunhyang University Cheonan Hospital, Cheonan, Republic of Korea \\ ${ }^{3}$ Department of Biostatistics, Soonchunhyang University Cheonan Hospital, Cheonan, Republic of Korea \\ ${ }^{4}$ Institute of Tissue Regeneration, College of Medicine, Soonchunhyang University, Cheonan, Republic of Korea \\ ${ }^{5}$ Soonchunhyang Institute of Medi-bio Science, Soonchunhyang University, Cheonan, Republic of Korea
}

Background: Proteinuria is a significant risk factor for progression of IgA nephropathy (IgAN) and has a positive correlation with severity of foot process effacement (FPE). We evaluated the relationship of FPE with proteinuria and histologic characteristics, including the Oxford classification.

Methods: Patients who underwent renal biopsy and were diagnosed with IgAN at a single center were retrospectively reviewed. Patients aged less than 18 years and those with the possibility of secondary causes were excluded from the study. Subsequently, we evaluated the association between degree of proteinuria, severity of FPE, and histologic characteristics, including the Oxford classification and other immunofluorescence stains.

Results: A total of 805 cases of renal biopsy was performed at our institution, and 327 patients were diagnosed with IgAN. Among them, 82 patients were excluded. Severity of FPE had an impact on the degree of proteinuria. Notably, the group with diffuse FPE had more than about $1.3 \mathrm{~g} /$ day of urine protein compared to those with rare FPE. Among the histologic characteristics, M1 score and immune deposition of IgG affected severity of FPE (hazard ratios [95\% confidence interval], 1.90 [1.10 to 3.26], and 3.77 [1.66 to 8.54], respectively).

Conclusion: Severity of FPE had an impact on the degree of proteinuria and may be associated with the pathogenesis of IgAN.

Keywords: Glomerulonephritis, Immunoglobulin A, Nephropathy, Podocytes, Proteinuria

Received January 28, 2020; Revised June 1, 2020;

Accepted June 4, 2020

Editor: Jung Eun Lee, Sungkyunkwan University, Seoul, Republic of Korea

Correspondence: Samel Park

Department of Internal Medicine, Soonchunhyang University Cheonan Hospital, 31 Suncheonhyang 6-gil, Dongnam-gu, Cheonan 31151, Republic of Korea. E-mail: samelpark17@schmc. ac.kr

Copyright (c) 2020 by The Korean Society of Nephrology

(a) This is an open-access article distributed under the terms of the Creative Commons Attribution Non-Commercial License (http://creativecommons. org/licenses/by-nc-nd/4.0/), which permits unrestricted non-commercial use, distribution, and reproduction in any medium, provided the original work is properly cited.

\section{Introduction}

IgA nephropathy (IgAN) is the leading cause of primary chronic glomerular disease in the world [1,2]. A slow progression to end-stage renal disease over two decades has been reported in $30 \%$ of patients [3]. Diagnosis is based on presence of mesangial proliferation with dominant or co-dominant IgA deposition [4]. The deposition of circulating immune complex, caused by autoantibodies against galactose-deficient IgAl (Gd-IgAl), in glomerular mesangium is considered the emerging pathogenesis of IgAN [5]. The deposited immune complex consisting of Gd-IgAl and antibody (mostly IgG and partly IgA) leads 
to podocyte activation and injury through mesangialderived cytokines $[6,7]$.

Proteinuria has been reported to be the most important risk factor for progression of IgAN $[8,9]$. Approximately $10 \%$ of IgAN present with nephrotic syndrome and exhibit poor prognosis in absence of response to treatment [10]. Another study reports presence of extensive foot process effacement (FPE) in patients with IgAN with nephrotic syndrome, similar to minimal change disease [11]. In addition, proteinuria has a positive correlation with severity of FPE in IgAN [12]. However, the relationship of FPE and proteinuria or with other histologic characteristics, like the Oxford classification, has not been examined. Therefore, we evaluated the relationship between FPE, proteinuria, and histologic characteristics, including the Oxford classification.

\section{Methods}

\section{Study population}

This study was reviewed and approved by the Institutional Review Board of Soonchunhyang University Cheonan Hospital (Cheonan, Korea) (approval number: 2019-08-026-001). Patients who underwent a renal biopsy and were diagnosed with IgAN at Soonchunhyang University Cheonan Hospital (Cheonan, Korea) from January 2011 to December 2018 were evaluated. Adult patients ( $\geq 18$ years) with predominant immunofluorescence (IF) microscopy of IgA $(\geq 1+)$ in the glomerular mesangial area were enrolled. Patients with active cancer, positive serology for hepatitis virus antigens (B and C), acute infection, or history of diabetes \pm biopsy confirmed diabetic nephropathy were excluded to rule out secondary causes.
Patients without electron microscopy results that showed adequate glomerulus to evaluate severity of FPE were excluded. Patients with subendothelial electron-dense depositions were also removed from this study to exclude the interaction between subendothelial deposition and FPE. This study was conducted in accordance with the principles of the Declaration of Helsinki. Since this was a retrospective study, the requirement of informed consent was waived.

\section{Characteristics of renal pathology}

Diagnosis of IgAN was based on light and IF microscopy results by two expert pathologists (J.H. Lee and S.H. Jang). The histologic features were described according to the Oxford classification of mesangial hypercellularity (M, Fig. 1A), endocapillary hypercellularity (E, Fig. 1B), segmental glomerulosclerosis (S), tubular atrophy/ interstitial fibrosis (T), and crescents (C) [13-15]. Severity of FPE was determined based on only extent of FPE,
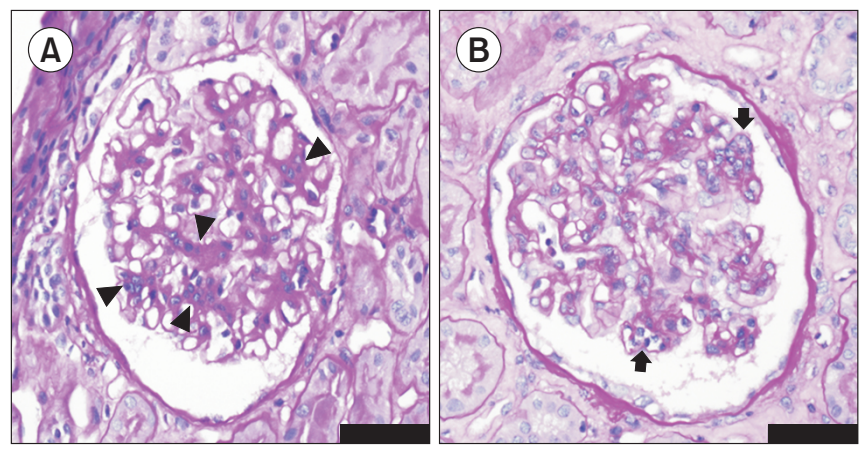

Figure 1. Representative histology by light microscopy of glomeruli with (A) mesangial hypercellularity and (B) endocapillary hypercellularity. Black bar represents $50 \mu \mathrm{m}$; black arrowheads, mesangial hypercellularity; black arrows, endocapillary hypercellularity.
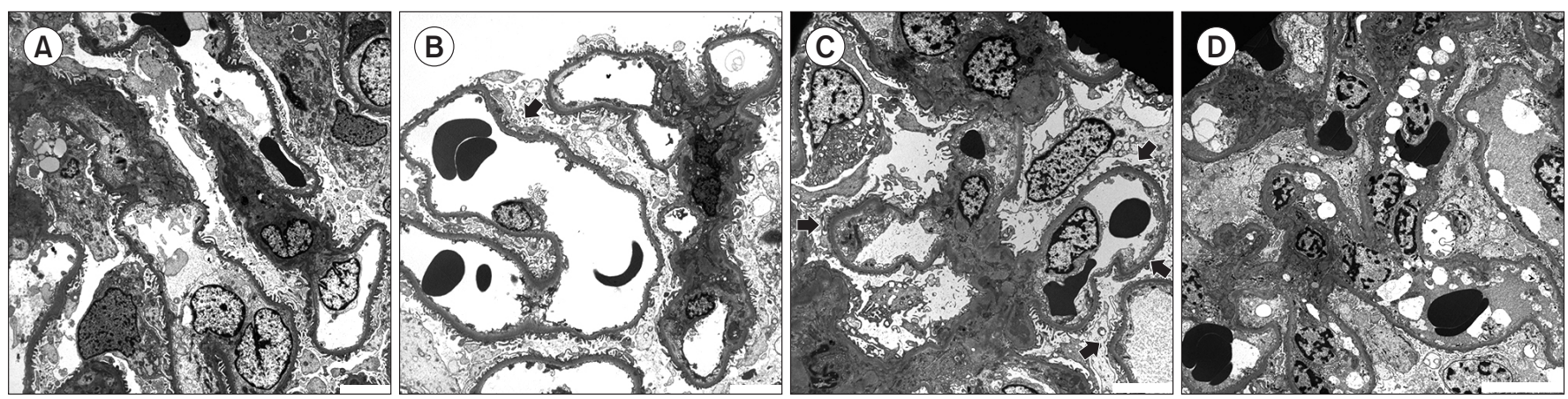

Figure 2. Representative images of electron microscopy of (A) rare, (B) mild, (C) moderate, and (D) diffuse foot process effacement. White bar represents $5 \mu \mathrm{m}$ in (A), (B), and (C) but $10 \mu \mathrm{m}$ in (D). Black arrows represent lesion with foot process effacement. 
which was established by visual inspection [16] using a semi-quantitative method. When more than $90 \%$ of the capillaries exhibited FPE, we defined the severity as 'diffuse.' If less than $10 \%$ of the capillaries exhibited FPE, it was designated as 'rare.' If FPE was observed in more than half (but not exceeding $90 \%$ ) of the total glomerular capillary length, it was defined as 'moderate'; if not (i.e., FPE was observed in less than half of the total glomerular capillary length), it was described as 'mild' (Fig. 2). The degree of IgA deposition was categorized as $1+, 2+$, and $\geq 3+$ (representing $3+$ and $4+$ ). Co-deposition of complement 3 (C3) was described as negative, trace, $1+$, and $\geq$ $2+$ (representing $2+$ and $3+$ ) and of IgG as a dichotomous level, negative or positive. Patients with number of globally sclerotic glomeruli (GSG) greater than the upper reference limit (95th percentile) of those expected on biopsy

Table 1. Clinical characteristics of patients with IgA nephropathy stratified according to foot process effacement $(n=245)$

\begin{tabular}{|c|c|c|c|c|c|}
\hline Characteristic & Rare FPE $(n=46)$ & Mild FPE $(n=111)$ & Moderate FPE $(n=67)$ & Diffuse FPE $(n=21)$ & $P$ value \\
\hline Age (yr) & $33(23-49)$ & $38(30-48)$ & $40(31-46)$ & $39(31-49)$ & 0.655 \\
\hline Male & $30(65.2)$ & $64(57.7)$ & $36(53.7)$ & 13 (61.9) & 0.656 \\
\hline HTN & $14(30.4)$ & $33(29.7)$ & $22(32.8)$ & $8(38.1)$ & 0.664 \\
\hline Current smoker & $9(19.6)$ & $20(18.0)$ & $9(13.4)$ & $3(14.3)$ & 0.799 \\
\hline $\mathrm{BMI}\left(\mathrm{kg} / \mathrm{m}^{2}\right)$ & $24.0(21.2-26.7)$ & $23.8(21.0-26.4)$ & $23.2(21.5-26.4)$ & $23.1(21.4-26.5)$ & 0.870 \\
\hline $\mathrm{MAP}(\mathrm{mmHg})$ & $89(76-97)$ & $93(83-97)$ & $93(83-97)$ & $93(82-107)$ & 0.403 \\
\hline WBC count $(/ \mu \mathrm{L})$ & 7,105 (5,933-8,165) & $6,710(5,820-8,120)$ & $7,080(5,590-8,720)$ & $6,640(5,630-8,405)$ & 0.823 \\
\hline Hemoglobin (g/dL) & $13.8 \pm 1.6$ & $13.7 \pm 1.6$ & $13.5 \pm 1.8$ & $12.8 \pm 2.6$ & $0.118^{\mathrm{a}}$ \\
\hline Platelet count $(/ \mu \mathrm{L})$ & $264(233-289)$ & $251(213-291)$ & $245(217-284)$ & $224(200-262)$ & 0.122 \\
\hline Protein (g/dL) & $6.9(6.5-7.2)$ & $6.8(6.3-7.2)$ & $6.7(6.2-7.2)$ & $6.1(4.8-6.9)$ & $0.002^{b}$ \\
\hline Albumin (g/dL) & $4.2(4.0-4.4)$ & $4.2(3.9-4.4)$ & $4.1(3.8-4.3)$ & $3.7(2.5-4.3)$ & $0.005^{b}$ \\
\hline BUN (mg/dL) & $13.8(11.1-16.1)$ & $14.5(12.6-17.8)$ & $15.7(12.2-19.3)$ & $16.7(12.3-27.5)$ & $0.064^{b}$ \\
\hline Creatinine $(\mathrm{mg} / \mathrm{dL})$ & $0.84(0.70-0.99)$ & $0.87(0.70-1.10)$ & $1.00(0.80-1.20)$ & $1.20(0.85-1.70)$ & $<0.001^{\mathrm{b}}$ \\
\hline eGFR $\left(\mathrm{mL} / \mathrm{min}\right.$ per $\left.1.73 \mathrm{~m}^{2}\right)$ & $103.5(93.2-123.4)$ & $108.0(77.8-122.3)$ & $88.4(64.5-112.6)$ & $71.1(37.2-107.9)$ & $<0.001^{\mathrm{b}}$ \\
\hline Uric acid (mg/dL) & $5.8(4.4-6.5)$ & $5.7(4.8-6.6)$ & $6.2(4.6-7.4)$ & $7.0(5.7-9.1)$ & $0.008^{b}$ \\
\hline Calcium (mg/dL) & $9.3(9.2-9.6)$ & $9.2(8.8-9.4)$ & $9.1(8.7-9.5)$ & $8.6(7.9-9.1)$ & $<0.001$ \\
\hline Corrected calcium (mg/dL) & $9.1 \pm 0.3$ & $9.0 \pm 0.4$ & $9.0 \pm 0.5$ & $8.9 \pm 0.5$ & 0.256 \\
\hline Phosphorus (mg/dL) & $3.5 \pm 0.5$ & $3.6 \pm 0.6$ & $3.5 \pm 0.5$ & $3.8 \pm 0.8$ & 0.218 \\
\hline Urine protein (g/day) & $0.38(0.16-0.70)$ & $0.60(0.35-1.25)$ & $0.60(0.33-1.45)$ & $0.74(0.38-2.70)$ & $0.003^{b}$ \\
\hline $\mathrm{PCR}(\mathrm{g} / \mathrm{g})$ & $0.30(0.15-0.52)$ & $0.55(0.28-1.13)$ & $0.58(0.34-1.12)$ & $1.22(0.29-2.21)$ & $<0.001^{\mathrm{b}}$ \\
\hline Total glomeruli (counts) & $32(18-42)$ & $23(16-35)$ & $22(13-34)$ & $22(14-26)$ & $0.038^{b}$ \\
\hline Glomerulosclerosis (\%) & $5.9(0.0-16.3)$ & $8.7(1.3-23.1)$ & $18.8(6.7-42.1)$ & $16.2(5.2-33.7)$ & $<0.001^{\mathrm{b}}$ \\
\hline GSG abnormal for age & $17(37.0)$ & $51(45.9)$ & $45(67.2)$ & $11(52.4)$ & $0.008^{b}$ \\
\hline \multicolumn{6}{|l|}{ Oxford classification } \\
\hline M1 & $14(30.4)$ & 49 (44.1) & $44(65.7)$ & $11(52.4)$ & $0.002^{b}$ \\
\hline E1 & $23(50.0)$ & $52(46.8)$ & $35(52.2)$ & $11(52.4)$ & 0.899 \\
\hline S1 & 34 (73.9) & $94(84.7)$ & $57(85.1)$ & $16(76.2)$ & 0.321 \\
\hline $\mathrm{T} 1$ & $4(8.7)$ & $25(22.5)$ & $19(28.4)$ & $6(28.6)$ & $0.009^{\mathrm{b}, \mathrm{c}}$ \\
\hline $\mathrm{T} 2$ & $0(0.0)$ & $2(1.8)$ & $6(9.0)$ & $0(0.0)$ & \\
\hline $\mathrm{C} 1$ & $13(28.3)$ & 37 (33.3) & $23(34.3)$ & $3(14.3)$ & $0.280^{\circ}$ \\
\hline $\mathrm{C} 2$ & $1(2.2)$ & $1(0.9)$ & $0(0.0)$ & $1(4.8)$ & \\
\hline
\end{tabular}

Data are presented as mean \pm standard deviation, median (interquartile range), or number (\%) as appropriate.

$\mathrm{BMI}$, body mass index; BUN, blood urea nitrogen; C, crescents; E, endocapillary hypercellularity; eGFR, estimated glomerular filtration rate; FPE, foot process effacement; GSG, globally sclerotic glomeruli; HTN, hypertension; M, mesangial hypercellularity; MAP, mean arterial pressure; PCR, urine protein to creatinine ratio; S, segmental glomerulosclerosis; T, tubular atrophy/interstitial fibrosis; WBC, white blood cell.

${ }^{a} P$ value was calculated using one-way ANOVA. In other cases, it was calculated using the Kruskal-Wallis test. ${ }^{b}$ Statistical significance in the trend test. For continuous variables, the Jonckheere-Terpstra test was used. For categorical variables, Cochran-Armitage (only for $2 \times \mathrm{k}$ tables) or linear-by-linear test was used. ${ }^{\circ}$ Fisher's exact test was used. 
according to age were grouped into GSG abnormal for age [17]. Patients aged $>77$ years were regarded to have the same thresholds as the 75 to 77 -years group because reference limits were not available for patients aged $>77$ years. Patients not assigned to the GSG abnormal group for age were grouped with GSG normal for age.

\section{Covariates}

The clinical and demographical characteristics of the patients, including age, sex, body mass index (BMI), history of hypertension, current smoking status, and blood pressure on admission day, were collected by reviewing electronic medical records. The patients' laboratory data, including complete blood count, serum protein, albumin, blood urea nitrogen, creatinine, uric acid, calcium, and phosphorus, were collected. Based on creatinine level, the estimated glomerular filtration rate (eGFR) was calculated using the Chronic Kidney Disease-Epidemiology Collaboration equation [18]. Twenty-four-hour collected urine protein and urine protein to creatinine ratio were calculated.

\section{Statistical analyses}

The patients were categorized into four groups accord-
A

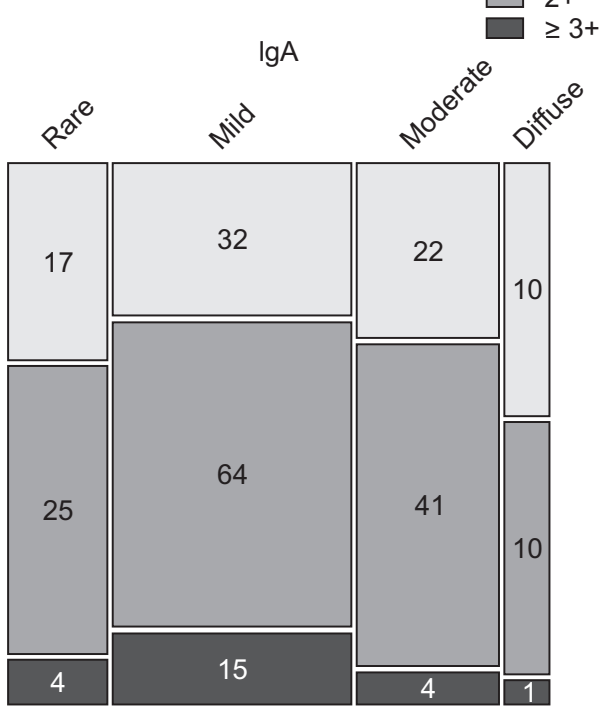

C
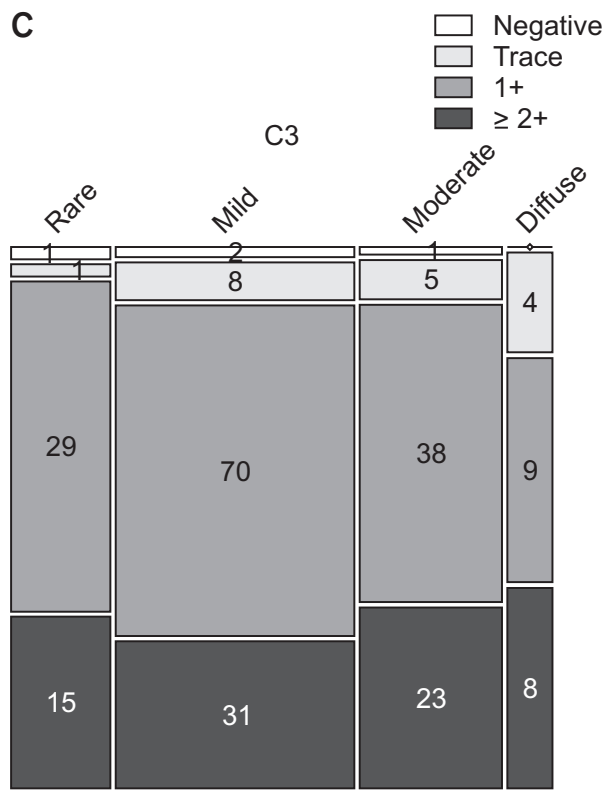

B
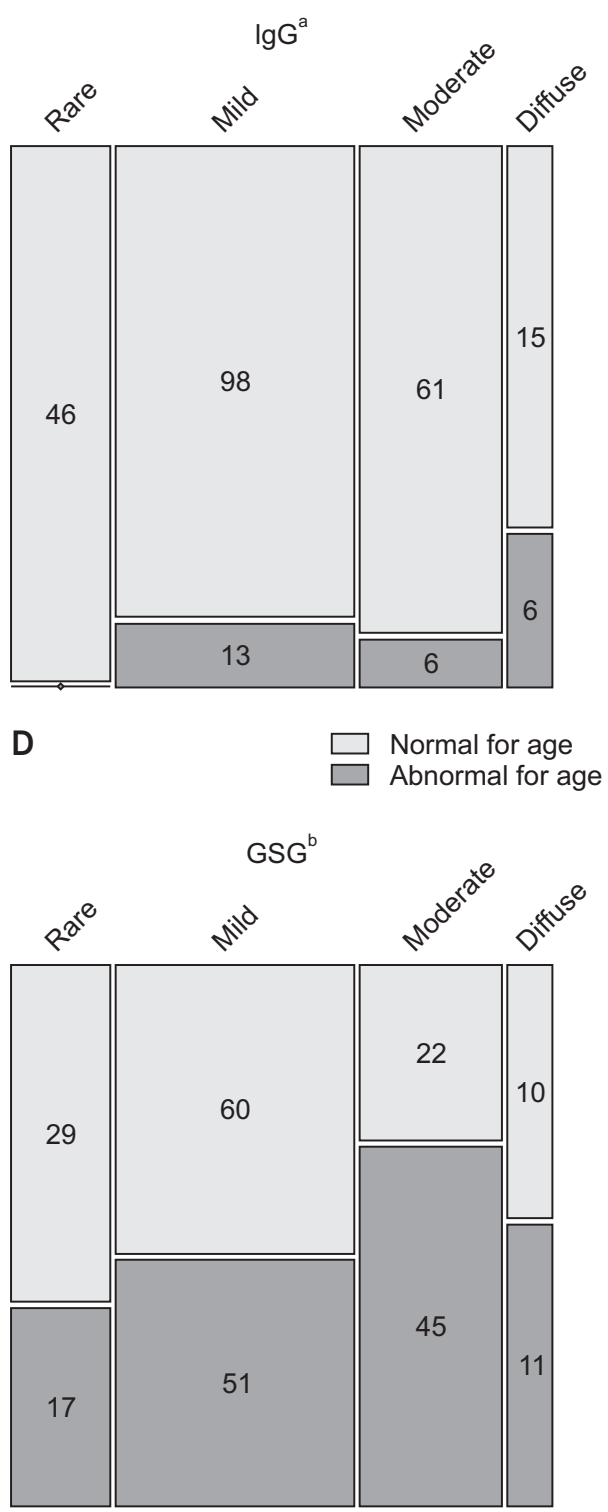

Figure 3. Characteristics of renal histology according to stratification of severity of foot process effacement. Represented based on $(A-C)$ immunofluorescence stain, (A) IgA, (B) IgG, (C), C3, and (D) globally sclerotic glomeruli (GSG) abnormal or normal for age. The numbers in the graph indicate the numbers of patients. Statistical significance in trend test; ${ }^{\mathrm{a}} P<0.01 ;{ }^{\mathrm{b}} P<0.05$. 
ing to severity of FPE: 1) rare, 2) mild, 3) moderate, and 4) diffuse. Categorical variables are presented as count (percentage) and continuous variables as mean \pm standard deviation or median (interquartile range) as appropriate. Comparisons between groups were performed using one-way ANOVA for normally distributed continuous variables and the Kruskal-Wallis test for non-normally distributed continuous variables. For categorical variables, Pearson's chi-square test or Fisher's exact test was performed. As a trend test, the Jonckheere-Terpstra test was used for continuous variables. For categorical variables, the Cochran-Armitage test was used in the case of $2 \times \mathrm{k}$ tables, while the linear-by-linear association test was used in other cases. To estimate the role of covariates in degree of proteinuria, generalized linear models were used. The basic model was created based on clinical information that was classically considered significant, including the Oxford classification. Then, a new model including severity of FPE was built to demonstrate the as- sociation between FPE and proteinuria. The impacts of the histologic characteristics on FPE were evaluated using ordinary logistic analysis because the parallelism test for ordinary logistic analysis was satisfied.

Statistical analyses were performed using IBM SPSS Statistics 25.0 for Windows (IBM Corp., Armonk, NY, USA) and R version 3.4.3 (The R Foundation for Statistical Computing, Vienna, Austria).

\section{Results}

A total of 805 cases of renal biopsy was performed at Soonchunhyang University Cheonan Hospital (Cheonan, Korea) from January 2011 to December 2018. Among them, 327 patients were diagnosed with IgAN. Overall, 82 patients were excluded from this study with the following causes: 8 , inadequate glomerulus in electron microscopy; 22 , age $<18$ years (range: 10 to 17 years); 7 , active cancer (1, cholangiocarcinoma; 1 , gastric cancer; 2 , gynecologic
A
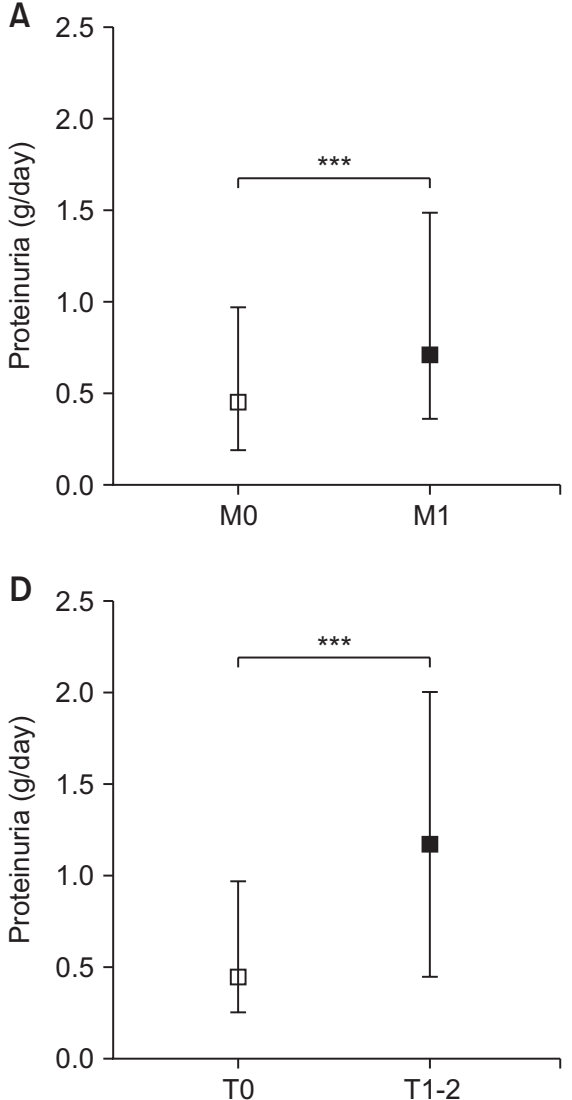
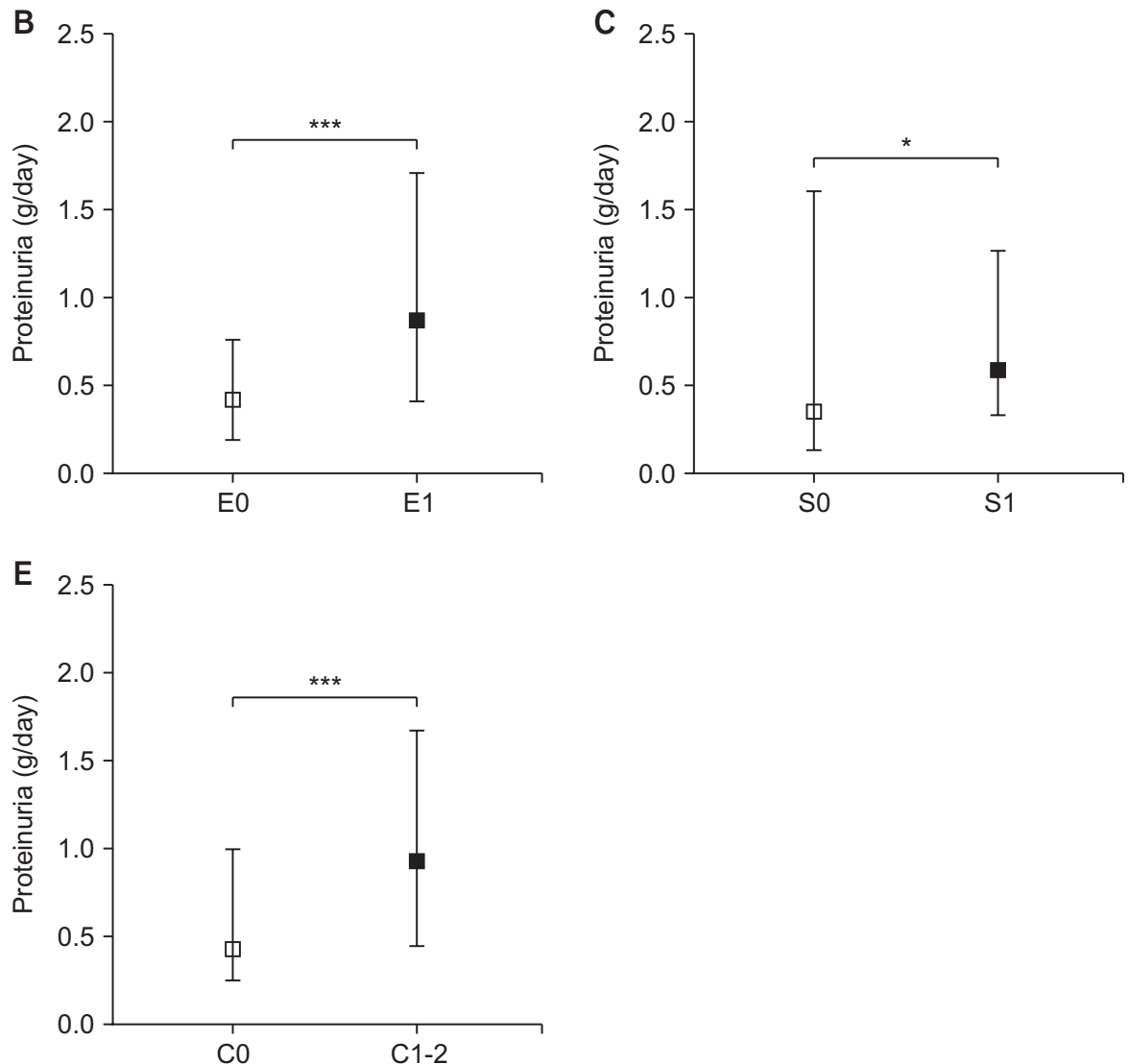

Figure 4. Degree of proteinuria based on the Oxford classification. (A) Mesangial hypercellularity, M; (B) endocapillary hypercellularity, E; (C) segmental glomerulosclerosis, S; (D) tubular atrophy/interstitial fibrosis, T; and (E) crescents, C. Presented as median and interquartile range; $* P<0.05 ; * * * P<0.001$. 
cancer; 1, thyroid cancer; 1, breast cancer; and 1, hepatocellular carcinoma); 15, hepatitis B antigen positive; 1 , hepatitis $C$ antigen positive; 1 , suspicion of infectionassociated IgAN; and 19, diabetes \pm concomitant diabetic nephropathy. To eliminate the interaction between subendothelial deposition and FPE, nine patients with subendothelial deposits were excluded. Finally, a total of 245 patients was analyzed.

Table 1 shows the clinical characteristics stratified according to severity of FPE. In patients with severe FPE, more urine protein, higher serum uric acid level, and lower serum albumin level were noted. Reduced renal function with higher $\mathrm{T}$ score in the Oxford classification was observed in the higher FPE group. Calcium level seemed to be lower in the more severe FPE group. However, there was no difference in corrected calcium level between the groups, implying an effect of serum albumin level. Additionally, the group with severe FPE had a more prevalent M1 score in the Oxford classification, a higher proportion of GSG abnormal for age, and greater deposition of IgG (Table 1, Fig. 3).

We evaluated the possible factors with an impact on de- gree of proteinuria. All scores of the Oxford classification exhibited a relationship with proteinuria (Fig. 4). Model 1 was built with variables that were classically considered significant, including age, male sex, history of hypertension, current smoking status, BMI, mean arterial pressure, eGFR, uric acid, and the Oxford classification (Table 2). The score of El was associated with more severe proteinuria (about $0.5 \mathrm{~g} /$ day more than E0). When severity of FPE was added to Model 1 (i.e., Model 2), it exerted an effect on proteinuria independently. Notably, patients with diffuse FPE had more than $1.30 \mathrm{~g} /$ day (0.63 to 1.96 $\mathrm{g}$ /day) of proteinuria compared to those with rare FPE, suggesting the significant role of FPE in proteinuria in patients with IgAN (Table 2). The histologic characteristics in correlation with severity of FPE were evaluated. As a result, severity of FPE was found to be associated with M1 in the Oxford classification and with deposition of IgG in IF (Table 3).

\section{Discussion}

Our results showed that severity of FPE had an impact

Table 2. The clinical and histologic characteristics associated with level of urine protein (g/day)

\begin{tabular}{|c|c|c|c|c|}
\hline \multirow{2}{*}{ Variable } & \multicolumn{2}{|l|}{ Model 1} & \multicolumn{2}{|l|}{ Model 2} \\
\hline & $\beta(95 \% \mathrm{Cl})$ & $P$ value & $\beta(95 \% \mathrm{Cl})$ & $P$ value \\
\hline Age, per 10 years & $-0.04(-0.19$ to 0.12$)$ & 0.655 & $-0.03(-0.18$ to 0.12$)$ & 0.713 \\
\hline Male & $0.20(-0.19$ to 0.60$)$ & 0.312 & $0.26(-0.12$ to 0.64$)$ & 0.185 \\
\hline HTN & $-0.08(-0.49$ to 0.32$)$ & 0.689 & $0.01(-0.39$ to 0.40$)$ & 0.977 \\
\hline Current smoker & $0.10(-0.34$ to 0.54$)$ & 0.651 & $0.13(-0.29$ to 0.56$)$ & 0.544 \\
\hline BMI, per $1 \mathrm{~kg} / \mathrm{m}^{2}$ & $0.04(-0.00$ to 0.09$)$ & 0.058 & 0.05 (0.01 to 0.09$)$ & 0.023 \\
\hline MAP, per $1 \mathrm{mmHg}$ & $0.02(0.00$ to 0.03$)$ & 0.011 & 0.01 (0.00 to 0.03$)$ & 0.032 \\
\hline eGFR, per $10 \mathrm{~mL} / \mathrm{min} / 1.73 \mathrm{~m}^{2}$ & $-0.12(-0.21$ to -0.03$)$ & 0.009 & $-0.09(-0.18$ to 0.00$)$ & 0.052 \\
\hline Uric acid, per mg/dL & -0.04 ( -0.16 to 0.09$)$ & 0.545 & -0.07 (-0.19 to 0.05$)$ & 0.271 \\
\hline \multicolumn{5}{|l|}{ Oxford classification } \\
\hline M1 (vs. M0) & $0.09(-0.25$ to 0.43$)$ & 0.621 & $0.04(-0.29$ to 0.38$)$ & 0.799 \\
\hline E1 (vs. E0) & 0.51 (0.16 to 0.87$)$ & 0.004 & 0.53 (0.19 to 0.87$)$ & 0.002 \\
\hline S1 (vs. S0) & $-0.38(-0.82$ to 0.05$)$ & 0.084 & $-0.40(-0.82$ to 0.03$)$ & 0.067 \\
\hline T1/2 (vs. T0) & $-0.02(-0.48$ to 0.44$)$ & 0.942 & $0.03(-0.43$ to 0.48$)$ & 0.912 \\
\hline C1/2 (vs. C0) & $0.08(-0.31$ to 0.46$)$ & 0.690 & $0.14(-0.23$ to 0.52$)$ & 0.457 \\
\hline \multicolumn{5}{|l|}{ Foot process effacement } \\
\hline Rare & & & Reference & \\
\hline Mild & & & 0.47 (0.05 to 0.89$)$ & 0.028 \\
\hline Moderate & & & $0.50(0.02$ to 0.97$)$ & 0.042 \\
\hline Diffuse & & & $1.30(0.63$ to 1.96$)$ & $<0.001$ \\
\hline
\end{tabular}

Multivariable generalized linear model was used $(n=245)$.

$\mathrm{BMI}$, body mass index; C, crescents; $\mathrm{Cl}$, confidence interval; E, endocapillary hypercellularity; eGFR, estimated glomerular filtration rate; HTN, hypertension; M, mesangial hypercellularity; MAP, mean arterial pressure; S, segmental glomerulosclerosis; T, tubular atrophy/interstitial fibrosis. 
on degree of proteinuria, and that mesangial proliferation and immune deposits of IgG were associated with severity of FPE. Considering the changes in actin dynamics induced by cytoskeleton rearrangements in podocyte, a connection between FPE and proteinuria was conceiv-

Table 3. The histologic characteristics associated with severity of foot process effacement

\begin{tabular}{lcc}
\hline \multicolumn{1}{c}{ Variable } & Odds ratios $(95 \% \mathrm{Cl})$ & $P$ value \\
\hline Oxford classification & & \\
M1 (vs. M0) & $1.90(1.10-3.26)$ & 0.021 \\
E1 (vs. E0) & $1.16(0.68-2.00)$ & 0.586 \\
S1 (vs. S0) & $1.10(0.55-2.20)$ & 0.781 \\
T1/2 (vs. T0) & $1.79(0.96-3.34)$ & 0.065 \\
C1/2 (vs. C0) & $0.68(0.38-1.23)$ & 0.206 \\
Immunofluorescence & & \\
IgA & Reference & \\
1+ & $0.90(0.49-1.65)$ & 0.737 \\
$2+$ & $0.52(0.20-1.32)$ & 0.170 \\
$\geq 3+$ & & \\
IgG & Reference & \\
Negative & $3.77(1.66-8.54)$ & 0.001 \\
Positive & & \\
C3 & Reference & \\
Negative & $2.09(0.26-16.94)$ & 0.489 \\
Trace & $0.88(0.13-6.21)$ & 0.901 \\
1+ & $1.12(0.14-8.79)$ & 0.912 \\
$\geq 2+$ & Reference & \\
Age-adjusted glomerulosclerosis & $0.89-2.79)$ & 0.122 \\
GSG normal for age & & \\
GSG abnormal for age & & \\
\hline
\end{tabular}

Multivariable ordinary logistic analysis was used $(n=245)$.

C, crescents; $\mathrm{Cl}$, confidence interval; E, endocapillary hypercellularity; GSG, globally sclerotic glomeruli; M, mesangial hypercellularity; S, segmental glomerulosclerosis; T, tubular atrophy/interstitial fibrosis. able [19]. Our results suggest that mesangial proliferation and deposition of IgG are linked with FPE of podocytes in IgAN.

In our cohort, all the Oxford classification scores exhibited a statistically significant relationship with proteinuria (Fig. 4). However, there were several discrepancies in the association between the Oxford classification and degree of proteinuria [20-22]. The discrepancies noted in previous studies may be attributed to severity of FPE.

In our final model, El in the Oxford classification and severity of FPE were associated with proteinuria (Table 2). Chakera et al [23] reported that E-lesions with no immunosuppressive treatment exhibited prognostic value. E score and degree of proteinuria were significantly related. However, there was no improvement of the goodness of fit when an interaction term between E-score and proteinuria was added [23]. Another study from China reported that crescents were more prevalent with E1 score [24]. Our result was consistent with this previous study. Their subsequent study showed that CD68 infiltrates were associated with E score, suggesting that the pathogenesis of more severe proteinuria in patients with E score was correlated with renal inflammation [25]. They also reported a significant association between $\mathrm{E}$ and $\mathrm{C}$ scores. In our cohort, a similar relationship between E and C scores was observed (Fig. 5A). Patients with E1 had more proteinuria than those with E0 irrespective of C score (Fig. 5B). Given that formation of crescents represented a nonspecific response to severe injury to the glomerular capillary wall and discontinuities of capillary walls resulting in leakage of inflammatory materials into Bowman's space [26,27], endocapillary hypercellularity may precede crescent formation.

Deposition of immune complexes composed of Gd-
A

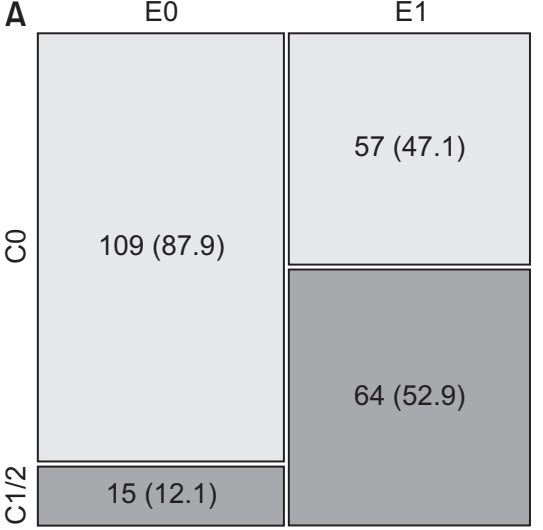

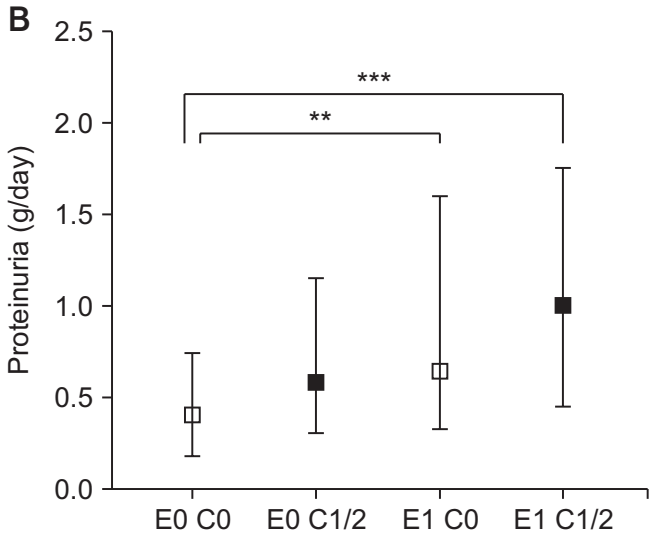

Figure 5. The association of endocapillary hypercellularity and crescents and their implications on proteinuria. (A) Crescents are more prevalent in endocapillary hypercellularity, $P<0.001$ by Pearson's chi-square test. (B) The amount of proteinuria is larger in endocapillary hypercellularity, irrespective of crescents. $* * P<0.01$; $* * * P<0.001$ by Kruskal-Wallis and Dunn's multiple comparisons test.

C, crescents; E, endocapillary hypercellularity. 
IgAl and its specific autoantibodies in the mesangial area have a pivotal role in pathogenesis of IgAN [28]. Deposition of immune complexes in the mesangial area leads to proliferation of mesangial cells, thereby affecting other cells in the nephron, i.e., podocytes and tubular epithelial cells [29]. Podocyte injury results in podocyte detachment, podocyte hypertrophy, and FPE [30]. Previous studies have shown that podocytopathy induced by podocyte loss correlated with disease severity in IgAN [31], leading to segmental sclerosis and disease progression $[32,33]$. Given that mesangial proliferation leads to podocyte injury in IgAN $[6,7]$, it was conceivable that mesangial hypercellularity was associated with severity of FPE, as shown in our results (Table 3 ).

Additionally, our study showed that patients with positive IgG deposits in IF have greater FPEs (Fig. 3, Table 3). Previous studies have demonstrated that patients with IgG deposition exhibited more detrimental renal outcomes [34-36]. Since there were several cases in which IgG deposits were not found in IF microscopy, it was considered that IgA autoantibodies specific to Gd-IgAl were generated [37]. However, in a recent study, Gd-IgAlspecific IgG autoantibodies were found even in patients with negative IgG by IF microscopy [38]. The antigenicity in IF could be masked by sampling errors that could occur during renal biopsy and by the three-dimensional structure of the glomeruli. Nonetheless, if any patient had numerous lesions with co-deposition of IgG in their glomeruli, those with positive IgG could be identified more easily in renal biopsy samples. Therefore, it was reasonable to assume that the more common is IgG deposition the more severe is the disease. Taken together, our results implied that IgG deposition induced podocyte injury and FPE.

Our study had several limitations. First, this was a retrospective study from a single center. Additionally, all patients involved in this study were Korean. Therefore, it may be problematic to apply our results to other races. Second, the severity of FPE was determined by visual inspection in a semi-quantitative fashion. Third, the proportion of IgG deposits in our cohort was smaller (28 cases, 10.4\%) compared to previous reports [34-36]. These results might be affected by a regional determinant of renal biopsies, such as medical insurance or tests for mandatory military service. Nevertheless, another study on deposition of IgG in Korea reported that $31.9 \%$ of cases had IgG deposition in the glomerulus [35]. This discrepancy could be a weakness of our study.

In conclusion, our results suggest that severity of FPE had an impact on degree of proteinuria and may be associated with pathogenesis of IgAN.

\section{Conflicts of interest}

All authors have no conflicts of interest to declare.

\section{Funding}

This research was supported by a grant (NRF-2019R1G1 A1099728) from the National Research Foundation (NRF) of Korea and a grant (No. 2019M3E5D1A02069071) from the Bio \& Medical Technology Development Program of the National Research Foundation (NRF) funded by the Korean government (MSIT). This study was also supported by the Soonchunhyang University Research Fund.

\section{Authors' contributions}

Ji-Hye Lee and Si-Hyong Jang reviewed biopsies. NamJun Cho, Nam Hun Heo, and Samel Park collected data and performed statistical analysis. Ji-Hye Lee and Samel Park conceptualized this study and drafted the manuscript. Hyo-Wook Gil, Eun Young Lee, and Jong-Seok Moon advised on the study.

\section{References}

[1] Wyatt RJ, Julian BA. IgA nephropathy. N Engl J Med 2013; 368:2402-2414.

[2] Tomino Y. Diagnosis and treatment of patients with IgA nephropathy in Japan. Kidney Res Clin Pract 2016;35:197-203.

[3] D'Amico G. Natural history of idiopathic IgA nephropathy and factors predictive of disease outcome. Semin Nephrol 2004;24:179-196.

[4] Roberts IS. Pathology of IgA nephropathy. Nat Rev Nephrol 2014;10:445-454.

[5] Robert T, Berthelot L, Cambier A, Rondeau E, Monteiro RC. Molecular insights into the pathogenesis of IgA nephropathy. Trends Mol Med 2015;21:762-775.

[6] Lai KN, Leung JC, Chan LY, et al. Activation of podocytes by mesangial-derived TNF-alpha: glomerulo-podocytic communication in IgA nephropathy. Am J Physiol Renal Physiol 
2008;294:F945-F955.

[7] Lai KN, Leung JC, Chan LY, et al. Podocyte injury induced by mesangial-derived cytokines in IgA nephropathy. Nephrol Dial Transplant 2009;24:62-72.

[8] Radford MG Jr, Donadio JV Jr, Bergstralh EJ, Grande JP. Predicting renal outcome in IgA nephropathy. J Am Soc Nephrol 1997;8:199-207.

[9] Reich HN, Troyanov S, Scholey JW, Cattran DC; Toronto Glomerulonephritis Registry. Remission of proteinuria improves prognosis in IgA nephropathy. J Am Soc Nephrol 2007;18:3177-3183.

[10] Kim JK, Kim JH, Lee SC, et al. Clinical features and outcomes of IgA nephropathy with nephrotic syndrome. Clin J Am Soc Nephrol 2012;7:427-436.

[11] Herlitz LC, Bomback AS, Stokes MB, Radhakrishnan J, D'Agati VD, Markowitz GS. IgA nephropathy with minimal change disease. Clin J Am Soc Nephrol 2014;9:1033-1039.

[12] Choi SY, Suh KS, Choi DE, Lim BJ. Morphometric analysis of podocyte foot process effacement in IgA nephropathy and its association with proteinuria. Ultrastruct Pathol 2010;34:195-198.

[13] Working Group of the International IgA Nephropathy Network and the Renal Pathology Society, Cattran DC, Coppo $\mathrm{R}$, et al. The Oxford classification of IgA nephropathy: rationale, clinicopathological correlations, and classification. Kidney Int 2009;76:534-545.

[14] Working Group of the International IgA Nephropathy Network and the Renal Pathology Society, Roberts IS, Cook HT, et al. The Oxford classification of IgA nephropathy: pathology definitions, correlations, and reproducibility. Kidney Int 2009;76:546-556.

[15] Trimarchi H, Barratt J, Cattran DC, et al.; IgAN Classification Working Group of the International IgA Nephropathy Network and the Renal Pathology Society; Conference Participants. Oxford classification of IgA nephropathy 2016: an update from the IgA Nephropathy Classification Working Group. Kidney Int 2017;91:1014-1021.

[16] Liang M, Zhang X, Zhou J, et al. Clinicopathological characteristics and renal outcomes in IgA nephropathy patients with nephrotic range proteinuria. Int J Clin Exp Pathol 2016;9:4531-4538.

[17] Kremers WK, Denic A, Lieske JC, et al. Distinguishing agerelated from disease-related glomerulosclerosis on kidney biopsy: the Aging Kidney Anatomy study. Nephrol Dial Transplant 2015;30:2034-2039.

[18] Levey AS, Stevens LA, Schmid CH, et al.; CKD-EPI (Chronic
Kidney Disease Epidemiology Collaboration). A new equation to estimate glomerular filtration rate. Ann Intern Med 2009;150:604-612.

[19] Perico L, Conti S, Benigni A, Remuzzi G. Podocyte-actin dynamics in health and disease. Nat Rev Nephrol 2016;12: 692-710.

[20] Alamartine E, Sauron C, Laurent B, Sury A, Seffert A, Mariat C. The use of the Oxford classification of IgA nephropathy to predict renal survival. Clin J Am Soc Nephrol 2011;6: 2384-2388.

[21] Herzenberg AM, Fogo AB, Reich HN, et al. Validation of the Oxford classification of IgA nephropathy. Kidney Int 2011; 80:310-317.

[22] Shi SF, Wang SX, Jiang L, et al. Pathologic predictors of renal outcome and therapeutic efficacy in IgA nephropathy: validation of the oxford classification. Clin J Am Soc Nephrol 2011;6:2175-2184.

[23] Chakera A, MacEwen C, Bellur SS, Chompuk LO, Lunn D, Roberts ISD. Prognostic value of endocapillary hypercellularity in IgA nephropathy patients with no immunosuppression. J Nephrol 2016;29:367-375.

[24] Peng W, Tang Y, Tan L, Qin W. Crescents and global glomerulosclerosis in Chinese IgA nephropathy patients: a five-year follow-up. Kidney Blood Press Res 2019;44:103112.

[25] Soares MF, Genitsch V, Chakera A, et al. Relationship between renal CD68+ infiltrates and the Oxford classification of IgA nephropathy. Histopathology 2019;74:629-637.

[26] Jennette JC. Rapidly progressive crescentic glomerulonephritis. Kidney Int 2003;63:1164-1177.

[27] Singh SK, Jeansson M, Quaggin SE. New insights into the pathogenesis of cellular crescents. Curr Opin Nephrol Hypertens 2011;20:258-262.

[28] Suzuki H, Kiryluk K, Novak J, et al. The pathophysiology of IgA nephropathy. J Am Soc Nephrol 2011;22:1795-1803.

[29] Floege J, Moura IC, Daha MR. New insights into the pathogenesis of IgA nephropathy. Semin Immunopathol 2014; 36:431-442.

[30] Kriz W, Shirato I, Nagata M, LeHir M, Lemley KV. The podocyte's response to stress: the enigma of foot process effacement. Am J Physiol Renal Physiol 2013;304:F333F347.

[31] Lemley KV, Lafayette RA, Safai M, et al. Podocytopenia and disease severity in IgA nephropathy. Kidney Int 2002;61: 1475-1485.

[32] Hill GS, Karoui KE, Karras A, et al. Focal segmental glo- 
merulosclerosis plays a major role in the progression of IgA nephropathy. I. Immunohistochemical studies. Kidney Int 2011;79:635-642.

[33] El Karoui K, Hill GS, Karras A, et al. Focal segmental glomerulosclerosis plays a major role in the progression of IgA nephropathy. II. Light microscopic and clinical studies. Kidney Int 2011;79:643-654.

[34] Bellur SS, Troyanov S, Cook HT, Roberts IS; Working Group of International IgA Nephropathy Network and Renal Pathology Society. Immunostaining findings in IgA nephropathy: correlation with histology and clinical outcome in the Oxford classification patient cohort. Nephrol Dial Transplant 2011;26:2533-2536.
[35] Shin DH, Lim BJ, Han IM, et al. Glomerular IgG deposition predicts renal outcome in patients with IgA nephropathy. Mod Pathol 2016;29:743-752.

[36] Wada Y, Ogata H, Takeshige Y, et al. Clinical significance of IgG deposition in the glomerular mesangial area in patients with IgA nephropathy. Clin Exp Nephrol 2013;17:73-82.

[37] Knoppova B, Reily C, Maillard N, et al. The origin and activities of IgA1-containing immune complexes in IgA nephropathy. Front Immunol 2016;7:117.

[38] Rizk DV, Saha MK, Hall S, et al. Glomerular immunodeposits of patients with IgA nephropathy are enriched for IgG autoantibodies specific for galactose-deficient IgA1. J Am Soc Nephrol 2019;30:2017-2026. 